\title{
Empoderamiento de personas con discapacidad a través del aprendizaje colaborativo: proyecto Idipower
}

\author{
Empowering disabled persons through \\ collaborative learning: the Idipower project
}

\section{Palabras clave}

Aprendizaje colaborativo, modelo de apoyo, empoderamiento, participación.

\section{Keywords}

Collaborative learning, support model, empowerment, participation.

\section{Introducción}

Uno de los aspectos fundamentales en el ámbito de la discapacidad es saber escuchar a la persona, conocer lo que realmente quiere para apoyarla y acompañarla tanto en su día a día como en la consecución de sus objetivos de vida, evitando que otra persona imponga su criterio y tome decisiones por ella.

La forma de lograrlo es empoderar a la persona a través del desarrollo de sus capacidades, ofreciéndola el apoyo necesario para trazar su itinerario de vida a través del ejercicio y defensa de sus derechos y la promoción de su autonomía e independencia en todas y cada una de las áreas de su vida.

Sin duda alguna, el aprendizaje colaborativo es una herramienta pedagógica fundamental para el desarrollo y transferencia del conocimiento, si lo entendemos como un proceso que permite que todas las personas (personas con discapacidad, familias y profesionales) tengan la oportunidad de intercambiar opiniones, conocimientos y experiencias desde un mismo plano y con un mismo lenguaje, buscando soluciones prácticas para convertir a la persona con discapacidad en la protagonista de su propia vida. El aprendizaje colaborativo proporciona
Ma José Cabo González

<mjcabo@amica.es>

Pedagoga y directora técnica, asociación AMICA

Irene de los Ríos Gutiérrez <irened@amica.es>

Pedagoga, asociación AMICA

Para citar:

Cabo, M. J. y De los Ríos, I. (2016): "Empoderamiento de personas con discapacidad a través del aprendizaje colaborativo: proyecto Idipower". Revista Española de Discapacidad, 4 (2): $235-240$.

Doi: <https://doi.org/IO.5569/23405 I04.04.02.I3> 
"herramientas de actitud crítica, emancipada y transformativa en relación a la representación individual y colectiva de la realidad" (Albalá, 2016).

Ante esta situación de partida, surge la iniciativa de poner en marcha el Proyecto Idipower: empoderamiento de las personas con discapacidad a través del aprendizaje colaborativo, con la finalidad de desarrollar una metodología basada en la participación y la colaboración de las personas que configuran la red de apoyos a las personas con discapacidad, implicándose en el proceso de superar las barreras que impiden que la persona sea la protagonista de su propia vida y pueda tomar sus propias decisiones, estableciendo unos factores de éxito que favorezcan la inclusión y la participación a la comunidad como un derecho fundamental de las personas, el apoyo y acompañamiento en su entorno y el ejercicio de los derechos.

Un consorcio multinacional compuesto por cinco entidades dedicadas a apoyar a las personas con discapacidad: Amica, coordinadora del proyecto y coordinadora de centros ocupacionales de la Comunidad Valenciana (Copava, España), Asociación Croata de Terapeutas Ocupacionales (HURT, Croacia), Cercioeiras (Portugal) y CUDV Draga (Eslovenia) y un centro tecnológico especializado en metodologías de aprendizaje colaborativo y en el desarrollo de aplicación de tecnología para facilitar la puesta en práctica, Institut arbait und Technik (IAT, Alemania), han podido aunar sus fuerzas gracias a la financiación del programa Erasmus+ [2014-2016], dentro de la acción clave sobre Cooperación para la innovación y el intercambio de buenas prácticas: asociaciones estratégicas orientadas a la educación de adultos.

Fases de desarrollo

El proyecto Idipower se ha estructurado en cuatro fases que han dado lugar al desarrollo de cuatro productos finales:
- I I fase: determinación de los factores de éxito del modelo de apoyo a la persona.

Un total de 267 personas (personas con discapacidad, familias y profesionales) participaron en dos sesiones de trabajo desarrolladas en cada entidad donde tuvieron la oportunidad de compartir sus experiencias y vivencias, identificando y consensuando así los factores de éxito y buenas prácticas para la promoción de la autonomía e independencia de la persona. Las conclusiones de este proceso de análisis quedaron recogidas en el Informe sobre buenas prácticas para la implementación del modelo de apoyo a las personas con discapacidad.

- $\quad 2^{a}$ fase: diseño del programa de aprendizaje colaborativo Idipower.

Partiendo de las conclusiones obtenidas en la primera fase del proyecto, se desarrolló un programa formativo con los siguientes objetivos:

I. Sensibilizar en el trabajo conjunto a personas con discapacidad, familiares y profesionales para la planificación, ejecución y evaluación de apoyos individualizados, fundamentados en las buenas prácticas que faciliten la autonomía y el proyecto personal de la persona con discapacidad.

2. Proporcionar a todos los participantes conocimientos teóricos y prácticos sobre modelos de apoyo que favorezcan a las personas con discapacidad en torno a cuatro ejes: el ejercicio de derechos, la participación comunitaria, la vida independiente y el proyecto personal.

3. Proporcionar formación basada en un conjunto amplio de estrategias de acompañamiento vinculadas al modelo de calidad de vida centrada en la persona con necesidades complejas de apoyo, que sean de fácil aplicabilidad 
y que nos permitan desarrollar una metodología de acompañamiento coherente, desde un punto de vista ético, convirtiéndose en referencia en el ámbito de la atención, estimulación y apoyo a las personas con discapacidad.

- $\quad 3^{\text {a }}$ fase: desarrollo de los materiales formativos.

Teniendo en cuenta los objetivos del programa formativo y los factores de éxito y buenas prácticas para el desarrollo de un modelo de apoyo a la persona, se desarrollaron los materiales formativos.

En este proceso, una vez más, el trabajo colaborativo fue un elemento fundamental, dado que 128 personas tuvieron la oportunidad de participar en un proceso de validación de los materiales. A través de diversas sesiones, pudieron desarrollar una actitud crítica basada en el diálogo y el consenso, valorando los materiales, proponiendo cambios respecto a los contenidos, formato, accesibilidad, ilustraciones, tipo de letra... Cada una de estas cuestiones fue analizada detenidamente y fueron tenidas en cuenta a la hora de realizar mejoras posteriores.

- 4 a fase: desarrollo de la plataforma de aprendizaje colaborativo.

Todos los materiales han quedado expuestos en la plataforma Idipower (http:// community.idipower.eu/), diferenciados en dos niveles de información: el nivel básico, dirigido a todas aquellas personas que quieren iniciarse en las cuestiones tratadas o que tengan mayores dificultades de comprensión, y el nivel avanzado, dirigido a las personas que quieran profundizar en mayor medida en las diferentes áreas de conocimiento.

\section{Ilustración 1: plataforma de aprendizaje colaborativo Idipower.}

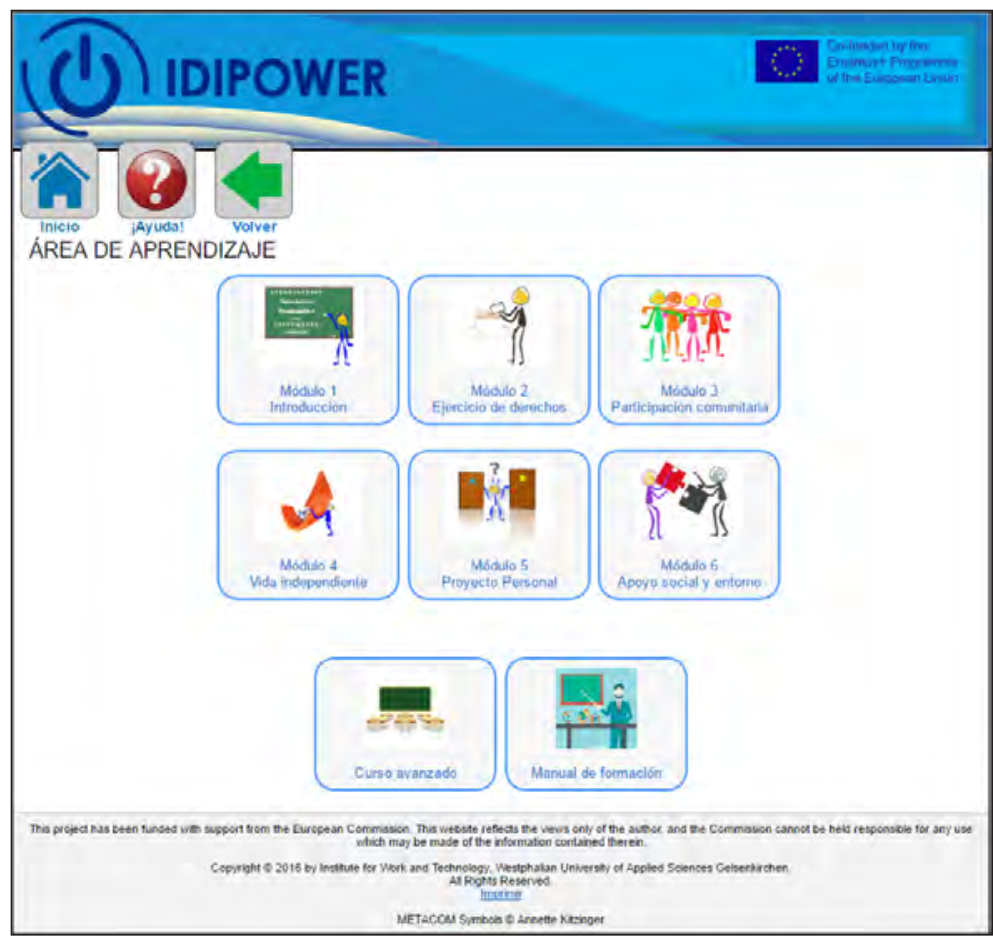


La plataforma Idipower permite:

- Conocer, analizar y reflexionar sobre los ejes principales que configuran la vida de una persona, los cuales se han estructurado en las siguientes dimensiones o categorías: calidad de vida, ejercicio de derechos, participación comunitaria, vida independiente, proyecto de vida y actitudes de las personas de apoyo.

- Compartir experiencias a través de diferentes espacios como el rincón de las historias.

- Debatir y expresar opiniones libremente a través de los temas propuestos en el foro.

$\mathrm{Al}$ igual que los materiales, la plataforma Idipower ha pasado por una fase de validación en las que las personas han participado para valorar y proponer acciones de mejora. La intención fundamental de la plataforma Idipower es ofrecer una herramienta sencilla pero con carácter transformador que tenga el poder de configurar una red de apoyo capaz de reflexionar y promover cambios de forma paulatina en la manera de apoyar a las personas, aprendiendo a escucharlas para saber lo que necesitan en cada momento.

\section{Resultados}

El desarrollo del proyecto Idipower ha sido una experiencia enriquecedora, no solo por los productos desarrollados, sino por los resultados alcanzados en las personas.

Todas las personas que han participado en el desarrollo de la metodología dentro del proyecto Idipower han valorado muy positivamente este proceso. Por vez primera, algunas personas con discapacidad han tenido la oportunidad de dar a conocer a los demás sus experiencias y sus necesidades. Se ha creado un espacio donde las personas pueden expresarse libremente y ser escuchadas, manifestándose con naturalidad, mostrando sus capacidades, compartiendo sus vivencias. Esta circunstancia incrementa el nivel de autoestima y la motivación así como el interés por participar.

De igual manera, para algunas familias ha sido la primera vez que han participado en un proceso de estas características. Estas personas han podido debatir, reflexionar y compartir experiencias, pero también han podido comprobar cómo a través de esta metodología su familiar adquiere mayor grado de independencia en la forma de expresarse y transmitir conocimiento, siendo capaz de reivindicar sus derechos y, sobre todo, su protagonismo hablando en primera persona.

El desarrollo de actitudes positivas hacia el trabajo es un elemento implícito en el proceso de aprendizaje colaborativo, así como el desarrollo de actitudes de cooperación y colaboración dentro del grupo para alcanzar un fin común.

Independientemente de las capacidades, "todas las personas desempeñan un papel fundamental dentro del grupo de trabajo".

Con apoyo o sin él, "todas las personas tienen ideas y opiniones que aportar", "todas las personas tienen experiencias de vida" y "todas las personas tienen deseos de alcanzar metas”.

La participación en este proceso ha promovido la toma de decisiones sobre diferentes aspectos: nivel de participación e implicación, aspectos técnicos del programa (contenidos, diseño, formato...) y a su vez ha favorecido el desarrollo de un pensamiento crítico.

Incluso la colaboración entre entidades de diferentes países de la Unión Europea ha generado un mayor interés en algunos de los participantes en mejorar sus competencias lingüísticas, con la intención de aumentar el nivel de comprensión de los mensajes y la necesidad de relacionarse con el resto de personas que forman parte del proyecto. 


\section{Conclusión}

Las seis entidades participantes han podido observar el clima de entendimiento que se produce cuando las personas con discapacidad, familias y profesionales se sitúan en un mismo nivel para tratar las mismas cuestiones. Conocer cómo se siente la otra persona, cuáles son sus inquietudes y necesidades es primordial para poder avanzar conjuntamente en la consecución de objetivos de vida.

La incorporación de esta metodología de participación y consenso dentro de las organizaciones, en el día a día, supone una transformación en el modelo de apoyo a las personas. Permite que los aprendizajes y los conocimientos se adquieran a través de las experiencias de las otras personas. El aprendizaje colaborativo se convierte en una herramienta de trabajo que sirve para derribar barreras físicas y psicológicas, compartir miedos e inquietudes pero, sobre todo, para avanzar en el desarrollo de nuevas experiencias, desarrollando estrategias de apoyo para las personas en su vida diaria, a lo largo de las diferentes etapas de su vida.

Para avanzar necesitamos compartir conocimiento y buenas prácticas que nos permitan aprender unas personas de otras independientemente de si tienen una discapacidad o no... intercambiando el saber hacer y desarrollando una buena transferencia entre todas las personas implicadas. Sin duda alguna, esta es la razón de ser el proyecto Idipower: aprender juntos, escuchando a las personas lo que quieren y apoyándolas en este proceso. 


\section{Referencias bibliográficas}

Aaidd (20II): Discapacidad Intelectual: Definición, clasificación y sistemas de Apoyo ( I I ${ }^{a}$ ed.), Madrid: Alianza Editorial.

Albalá, C. (20I6): Ocho puntos clave del aprendizaje colaborativo (en línea). <http:// ined2 I.com/ocho-puntos-clave-del-aprendizajecolaborativo>, accesoi I de julio de 2016.

Amica (2009): Descubriendo capacidades: Principios y valores que unen voluntades (en línea). <http://intranet.amica.es/publico/ webamica/o5 Comunicacion/LibrosPublicacione s/25aniversarioDescubriendoCapacidades.pdf>, acceso I 2 de septiembre de 2016.

Amica (20II). 20 años creando oportunidades de empleo (en línea). <http://intranet.amica.es/ publico/webamica/o 5 Comunicacion/LibrosPubl icaciones/2oaniversarioSOEMCAcreandoOport unidadesEmpleo.pdf $>$, acceso I 2 de septiembre de 2016.

Breipohl, W. et al. (20I I): "Mentoring people with disabilities". Optimum Q, 22 (3/4): 33-39.

Castillo, T. (20I3): Avanzar en mi independencia: El derecho a la autonomía personal, Torrelavega: Amica.
Castillo T. (2009): Aprendiendo a vivir. La enfermedad: descubrir las posibilidades que hay en mí, Barcelona: Ceac.

Castillo, T. (2007): Let me try! Disability: Towards a creative view of the human limitations, Barcelona: Ceac.

European Agency (20I4). Model policy for inclusive ICTs in education for persons with disabilities (en línea). <https://www.europeanagency.org/sites/default/files/UNESCO$\mathrm{G}_{3}$ ict $\%$ 20Model $\%$ 20Policy \% 200n \% 20 Inclusive $\%$ 20ICTs $\%$ 2 ofor $\%$ 2 2 Education $\% 20$ 4-20I4.pdf>, acceso I2 de septiembre de 2016.

Hamburg, I. y Bucksch, S. (2016): "Inclusive educational technology". Archieves of business research, 4 (I): 27-35.

Hamburg, I. y Bucksch, S. (2OI 5): "ICTbased approaches to support learners with disabilities". Journal of educational policy and entrepreneurial research (JEPER), 2 (6): I-I 2.

WHO (2OI I). International Classification of Functioning, Disability and Health (en línea). $<$ http://www.who.int/classifications/icd>, acceso I 2 septiembre de 2016 . 